\title{
TOPOLOGICAL REPRESENTATION FOR MONADIC IMPLICATION ALGEBRAS
}

\author{
M. ABAD, C. R. CIMADAMORE AND J. P. DÍAZ VARELA
}

\begin{abstract}
In this paper, every monadic implication algebra is represented as a union of a unique family of monadic filters of a suitable monadic Boolean algebra. Inspired by this representation, we introduce the notion of a monadic implication space, we give a topological representation for monadic implication algebras and we prove a dual equivalence between the category of monadic implication algebras and the category of monadic implication spaces.
\end{abstract}

\section{INTRODUCTION}

Implicative structures are particularly common among algebras associated with logical systems, although they arise in many other areas of mathematics. In general, they consist of an ordered set in which the ordering is characterized by a binary operation of implication $\rightarrow$. If the partial order is a semilattice order we have the Brouwerian semilattices, that are the models of the $\{\wedge, \rightarrow\}$-fragment of the intuitionistic propositional calculus. If the semilattice satisfies the property that every filter $[p]$ is a Boolean algebra, we obtain the implication algebras introduced by J. C. Abbot [4] - the variety of $\{\rightarrow\}$-subreducts of Boolean algebras. The variety of implication algebras is the unique atom in the lattice of subvarieties of the variety of Hilbert algebras, and more generally, the unique atom in the lattices of subvarieties of the variety of BCK-algebras. They can be characterized as the semisimple Hilbert algebras [4].

Monadic implication algebras were introduced by L. Iturrioz and A. Monteiro in [10] as a generalization of the notion of monadic Boolean algebras. These algebras were called monadic Tarski algebras in that work, and they are the variety of $\{\forall, \rightarrow\}$-subreducts ([1]) of monadic Boolean algebras, that is, they correspond to the monadic implicative fragment of the one-variable predicate superintuitionistic logics. In [1], M. Abad et al. determined the free monadic implication algebra with a finite set of free generators and in [2] the authors studied the lattices of varieties and quasivarieties of the variety of monadic implication algebras. The aim of this paper is to give a topological representation for monadic implication algebras. The main tool we use in the proofs of this result is the

2000 Mathematics Subject Classification. Primary: 06E15, 03G25, Secondary: 06F99.

Key words and phrases. Implication algebra, monadic Boolean algebra, implication spaces, dual categorical equivalence.

This paper is partially supported by Universidad Nacional del Sur and CONICET. 
duality between implication algebras and certain topological spaces, developed in [3]. The main definitions and the results of this duality are summarized in $§ 2.2$.

\section{Preliminaries}

If $X$ is an ordered set and $Y \subseteq X$, we say that $Y$ is an increasing set if, whenever $y \in Y, x \in X$ and $x \geq y$, we have $x \in Y$. Recall that a subset $C$ of a Boolean algebra B satisfies the finite meet property ( $\mathrm{fmp}$ for short), if 0 cannot be obtained with finite meets of elements of $C$, that is, the filter generated by $C$ in $\mathbf{B}$ is proper. If $\mathbf{B}$ is a Boolean algebra and $Y \subseteq B$, we denote by $F(Y)$ the filter generated by $Y$. We know that $F(Y)=\left\{b \in B: b \geq \bigwedge_{i=1}^{n} y_{i}, y_{i} \in Y, n \in \mathbb{N}\right\}$. If $Y$ is increasing, it is easy to prove that $F(Y)=\left\{b \in B: b=\bigwedge_{i=1}^{n} y_{i}, y_{i} \in X, n \in \mathbb{N}\right\}$. Proper maximal filters are called ultrafilters.

2.1. Duality for monadic Boolean algebras. We denote by $\mathrm{St}(\mathbf{B})$ the Stone space of a Boolean algebra $\mathbf{B}$, that is, the topological space of ultrafilters of $\mathbf{B}$ whose topology has as a base the family $\left\{N_{a}=\{U \in \operatorname{St}(\mathbf{B}): a \in U\}\right\}_{a \in B}$, and we denote by $\operatorname{Clop}(\mathbf{X})$ the Boolean algebra of all clopen subsets of a Boolean space $\mathbf{X}$. It is known (see $[11,5]$ ) that a dual equivalence between the category of Boolean algebras and Boolean homomorphisms and the category of Boolean spaces and continuous maps is given by

Theorem 2.1. [11] If $\boldsymbol{B}$ is a Boolean algebra, the map $\sigma_{\mathbf{B}}: \mathbf{B} \rightarrow \operatorname{Clop}(\operatorname{St}(\mathbf{B}))$ defined by $\sigma_{\boldsymbol{B}}(a)=N_{a}$ is a Boolean isomorphism, and if $\mathbf{X}$ is a Boolean space, the map $\varepsilon_{\mathbf{X}}: \mathbf{X} \rightarrow$ $\operatorname{St}(\operatorname{Clop}(\mathbf{X}))$ defined by $\varepsilon_{\mathbf{X}}(x)=\{U \in \operatorname{Clop}(\mathbf{X}): x \in U\}$ is a homeomorphism. Also, if $\boldsymbol{B}_{1}$ and $\boldsymbol{B}_{2}$ are Boolean algebras and $\alpha: B_{1} \rightarrow B_{2}$ is a homomorphism, the function $\operatorname{St}(h): \operatorname{St}\left(\mathbf{B}_{2}\right) \rightarrow \operatorname{St}\left(\mathbf{B}_{1}\right)$ defined by $\operatorname{St}(h)(U)=h^{-1}(U)$ is continuous. If $\mathbf{X}_{1}$ and $\mathbf{X}_{2}$ are Boolean spaces and $f: \mathbf{X}_{1} \rightarrow \mathbf{X}_{2}$ is continuous, then the mapping $\operatorname{Clop}(f): \operatorname{Clop}\left(\mathbf{X}_{2}\right) \rightarrow$ $\operatorname{Clop}\left(\mathbf{X}_{1}\right)$ defined by $\operatorname{Clop}(f)(U)=f^{-1}(U)$, is a homomorphism.

P. R. Halmos established in [7] a duality between quantifiers on a Boolean algebra $\mathbf{B}$ and certain equivalence relations on the Stone space of $\mathbf{B}$. Cignoli showed in [6] that this duality depends on the lattice structure of the Boolean algebra rather than on the existence of complements. More precisely, he showed that there is a duality between quantifiers on a bounded distributive lattice $\mathbf{L}$ and certain equivalence relations on the Priestley space of $\mathbf{L}$, and that this duality reduces to that discovered by Halmos when restricted to Boolean algebras. This duality is constructed as follows.

Let $\mathbf{X}$ be a Boolean space and $R$ an equivalence relation defined on $\mathbf{X}$. If $C \subseteq X$, we denote by $R C$ the union of all the equivalence classes that contain an element of $C$. We say that a subset $C \subseteq X$ is saturated if $R C=C$.

Definition 2.2. A pair $(\mathbf{X}, R)$ is called a $\mathbf{B}$-space if $\mathbf{X}$ is a Boolean space and the relation $R$ defined on $\mathbf{X}$ satisfies

(BE1) the equivalence classes for $R$ are closed in $\mathbf{X}$, and

(BE2) if $C$ is clopen then $R C$ is clopen.

If $\left(\mathbf{X}_{1}, R_{1}\right)$ and $\left(\mathbf{X}_{2}, R_{2}\right)$ are two $\boldsymbol{B}$-spaces, a map $f:\left(\mathbf{X}_{1}, R_{1}\right) \rightarrow\left(\mathbf{X}_{2}, R_{2}\right)$ is a $\mathbf{B}$-morphism if $f$ is continuous and for every $V \in \operatorname{Clop}\left(\mathbf{X}_{2}\right)$ it satisfies $R_{1}\left(f^{-1}(V)\right)=f^{-1}\left(R_{2}(V)\right)$. 
Let $\mathbf{B}=\langle B, \wedge, \vee, \neg, \exists, 0,1\rangle$ be a monadic Boolean algebra [7]. Sometimes we denote a monadic Boolean algebra $\mathbf{B}$ by $\langle B, \exists\rangle$ for brevity. Let $\operatorname{St}(\mathbf{B})$ denote the Stone space of the Boolean reduct of $\mathbf{B}$. If we define the relation $R_{\exists}$ in $\operatorname{St}(\mathbf{B})$ by

$$
U R_{\exists} V \text { if and only if } U \cap \exists B=V \cap \exists B \text {, for } U, V \in \operatorname{St}(\mathbf{B}),
$$

where $\exists B=\{\exists x: x \in B\}$, then the pair $\left(\operatorname{St}(\mathbf{B}), R_{\exists}\right)$ is a $\mathbf{B}$-space. Conversely, if $(\mathbf{X}, R)$ is a B-space, then we can define a quantifier on $\operatorname{Clop}(\mathbf{X})$ as $\exists_{R}(N)=R N$, for all $N \in \operatorname{Clop}(\mathbf{X})$. Thus $\left\langle\operatorname{Clop}(\mathbf{X}), \exists_{R}\right\rangle$ is a monadic Boolean algebra. Also if $h:\left\langle\mathbf{B}_{1}, \exists_{1}\right\rangle \rightarrow\left\langle\mathbf{B}_{2}, \exists_{2}\right\rangle$ is a monadic homomorphism, then the continuous function $\operatorname{St}(h):\left(\operatorname{St}\left(\mathbf{B}_{2}\right), R_{\exists_{2}}\right) \rightarrow\left(\operatorname{St}\left(\mathbf{B}_{1}\right), R_{\exists_{1}}\right)$ defined by $\operatorname{St}(h)(U)=h^{-1}(U)$, is a $\mathbf{B}$-morphism. If $f:\left(\mathbf{X}_{1}, R_{1}\right) \rightarrow\left(\mathbf{X}_{2}, R_{2}\right)$ is a $\mathbf{B}$ morphism, then the Boolean homomorphism $\operatorname{Clop}(f):\left\langle\operatorname{Clop}\left(\mathbf{X}_{2}\right), \exists_{R_{2}}\right\rangle \rightarrow\left\langle\operatorname{Clop}\left(\mathbf{X}_{1}\right), \exists_{R_{1}}\right\rangle$ defined by $\operatorname{Clop}(f)(U)=f^{-1}(U)$, is a monadic homomorphism. Finally, $\sigma_{\mathbf{B}}:\langle\mathbf{B}, \exists\rangle \rightarrow$ $\left\langle\operatorname{Clop}(\operatorname{St}(\mathbf{B})), \exists_{R}\right\rangle$ is an isomorphism of monadic Boolean algebras and $\varepsilon_{\mathbf{X}}:(\mathbf{X}, R) \rightarrow$ $\left(\operatorname{St}(\operatorname{Clop}(\mathbf{X})), R_{\exists_{R}}\right)$ is a homeomorphism of $\mathbf{B}$-spaces, and $\sigma_{\mathbf{B}}$ and $\varepsilon_{\mathbf{X}}$ establish the duality.

Congruences on a Boolean algebra can be translated into some subsets of the corresponding Stone space. Indeed, there is a relationship between filters of a Boolean algebra $\mathbf{B}$ and closed subsets of its Boolean space. If $F$ is a filter of $\mathbf{B}$ then $C_{F}=\{U \in \operatorname{St}(\mathbf{B}): F \subseteq U\}$ is a closed subset of $\operatorname{St}(\mathbf{B})$ and the map $F \rightarrow C_{F}$ is an anti-isomorphism from the lattice of filters of $\mathbf{B}$ to the lattice of closed subsets of $\mathrm{St}(\mathbf{B})$. It is easy to see that in the case of monadic Boolean algebras, the monadic filters are in one-to-one correspondence with the closed subsets in $\operatorname{St}(\mathbf{B})$ such that $R C=C$.

2.2. Duality for implication algebras. M. Abad, J. P. Díaz Varela and A. Torrens gave in [3] a topological representation for implication algebras based on the Stone topological representation for Boolean algebras. We begin by explaining these results briefly.

Definition 2.3. An implication algebra is an algebra $\langle A, \rightarrow\rangle$ of type (2) that satisfies the equations

(T1) $(x \rightarrow y) \rightarrow x=x$,

(T2) $(x \rightarrow y) \rightarrow y=(y \rightarrow x) \rightarrow x$,

(T3) $x \rightarrow(y \rightarrow z)=y \rightarrow(x \rightarrow z)$.

In any implication algebra $\mathbf{A}, a \rightarrow a=b \rightarrow b$, for any $a, b \in A$. Then $a \rightarrow a$ is constant and is represented by 1 . The relation $a \leq b$ if and only if $a \rightarrow b=1$ is a partial order, called the natural order of $\mathbf{A}$ and 1 is its greatest element. Relative to this partial order, $A$ is a $\vee$-semilattice where the join is given by $a \vee b=(a \rightarrow b) \rightarrow b$. As first examples of implication algebras, we can consider Boolean algebras $\langle B, \wedge, \vee, \neg, 0,1\rangle$ where the implication is defined as $a \rightarrow b=\neg a \vee b$; then $\langle B, \rightarrow\rangle$ is an implication algebra. Conversely, if $\mathbf{A}$ is an implication algebra with least element 0 then $\mathbf{A}$ is a Boolean algebra, where the complement of the element $a \in A$ is $\neg a=a \rightarrow 0$ and the meet is $a \wedge b=\neg(b \rightarrow \neg a)$. Besides, for each $a \in A,[a)=\{x \in A: a \leq x\}$ is a Boolean algebra in which, for $b, c \geq a$, we have $b \wedge c=(b \rightarrow(c \rightarrow a)) \rightarrow a$ and $\neg b=b \rightarrow a$. Actually, every implication algebra is a join-semilattice with greatest element, such that for each element $a \in A,[a)$ is a Boolean algebra (see [4]). 
If $\mathbf{A}$ is an implication algebra, there is a Boolean algebra $\mathbf{B}$ such that $\mathbf{A}$ is an implication subalgebra of $\mathbf{B}$, that is, implication algebras are the $\{\rightarrow, 1\}$-subreducts of Boolean algebras (see [4, Theorem 17]). Let $B(\mathbf{A})$ be the Boolean subalgebra of $\mathbf{B}$ generated by $A$, and $F(\mathbf{A})$ the filter generated by $A$ in $B(\mathbf{A})$. The implication algebra $\mathbf{A}$ is increasing in $B(\mathbf{A})$, (see [3]), and consequently, $A$ is a union of filters of $B(\mathbf{A})$.

Consider the following Boolean algebra, called the Boolean closure of $\mathbf{A}$ :

$$
\mathrm{Bo}(\mathbf{A})=\left\{\begin{array}{cl}
B(\mathbf{A}) & \text { if } F(\mathbf{A}) \neq B(\mathbf{A}) \\
B(\mathbf{A}) \times\{0,1\} & \text { if } F(\mathbf{A})=B(\mathbf{A}) .
\end{array}\right.
$$

It has been proved in [3] that for any implication algebra $\mathbf{A}, \operatorname{Bo}(\mathbf{A})$ is the least, up to isomorphism, Boolean algebra in which the filter $F(\mathbf{A})$ is an ultrafilter. As two implication algebras may have the same Boolean closure, they are distinguished by means of the family of all maximal elements in the set of all filters of $\operatorname{Bo}(\mathbf{A})$ contained in $A$. We will denote this family by $\mathrm{M}(\mathbf{A})$.

Definition 2.4. [3] An implication space is a triple $(\mathbf{X}, u, \mathcal{C})$ such that

(E1) $\mathbf{X}$ is a Boolean space,

(E2) $u$ is a fixed element of $X$,

(E3) $\mathcal{C}$ is an antichain, with respect to inclusion, of closed sets of $X$ such that $\bigcap \mathcal{C}=\{u\}$,

(E4) if $C$ is a closed subset of $X$ such that for every clopen $N$ of $X, C \subseteq N$ implies

$D \in \mathcal{C}$ for some $D \subseteq N$, then there exists $D^{\prime} \in \mathcal{C}$ such that $D^{\prime} \subseteq C$.

A function $f: \mathbf{X}_{1} \rightarrow \mathbf{X}_{2}$ between two implication spaces $\left(\mathbf{X}_{1}, u_{1}, \mathcal{C}_{1}\right)$ and $\left(\mathbf{X}_{2}, u_{2}, \mathcal{C}_{2}\right)$ is $i$-continuous if $f$ is continuous, $f\left(u_{1}\right)=u_{2}$, and for all $C \in \mathcal{C}_{2}$ there is $D \in \mathcal{C}_{1}$ such that $D \subseteq f^{-1}[C]$.

We denote by $\mathcal{I}$ the category whose objects are implication algebras and whose arrows are implication homomorphisms, and by $\mathcal{X}$ the category with implication spaces as objects and $i$-continuous maps as arrows. If $\mathbf{A}$ is an object in $\mathcal{I}$, then

$$
\mathbb{X}(\mathbf{A}):=(\operatorname{St}(\operatorname{Clop}(\operatorname{Bo}(\mathbf{A}))), F(\mathbf{A}), \mathcal{C}(\mathbf{A})),
$$

is in $\mathcal{X}$, where $\mathcal{C}(\mathbf{A})=\left\{C_{F}: F \in \mathbf{M}(\mathbf{A})\right\}$ is the family of closed sets corresponding to $\mathbf{M}(\mathbf{A})$. For each implication homomorphism $f: \mathbf{A}_{1} \rightarrow \mathbf{A}_{2}$, there is a Boolean homomorphism $\hat{f}: \operatorname{Bo}\left(\mathbf{A}_{1}\right) \rightarrow \operatorname{Bo}\left(\mathbf{A}_{2}\right)$ (see [3, Corollary 2.3]). Then we define $\mathbb{X}(f):=$ $\operatorname{St}(\hat{f}): \operatorname{St}\left(\operatorname{Bo}\left(\mathbf{A}_{2}\right)\right) \rightarrow \operatorname{St}\left(\operatorname{Bo}\left(\mathbf{A}_{1}\right)\right)$ by $\operatorname{St}(\hat{f})(U)=\hat{f}^{-1}(U)$, which is $i$-continuous. Analogously, given an implication space $(\mathbf{X}, u, \mathcal{C})$, the algebra

$$
\mathbb{I}(\mathbf{X}):=\{N \in \operatorname{Clop}(\mathbf{X}): C \subseteq N \text { for some } C \in \mathcal{C}\}
$$

is an implication algebra, and for each $i$-continuous $h: \mathbf{X}_{1} \rightarrow \mathbf{X}_{2}$ between two implication spaces $\left(\mathbf{X}_{1}, u_{1}, \mathcal{C}_{1}\right)$ and $\left(\mathbf{X}_{2}, u_{2}, \mathcal{C}_{2}\right)$, the map $\mathbb{I}(h):=\operatorname{Clop}(h) \uparrow \mathbb{I}\left(\mathbf{X}_{2}\right): \mathbb{I}\left(\mathbf{X}_{2}\right) \rightarrow \mathbb{I}\left(\mathbf{X}_{1}\right)$ defines an implication homomorphism. Thus, $\mathbb{X}: \mathcal{I} \rightsquigarrow \mathcal{X}$ and $\mathbb{I}: \mathcal{X} \rightsquigarrow \mathcal{I}$ are contravariant functors. It follows that $\varsigma_{\mathbf{A}}: \mathbf{A} \rightarrow \mathbb{X}(\mathbf{A})$ defined by $\varsigma_{\mathbf{A}}(a)=N_{a}$ is a natural dual transformation between the functor $\mathbb{I X}$ and the functor $\operatorname{Id}_{\mathcal{I}}$, and that the mapping $\tau_{\mathbf{X}}: \mathbf{X} \rightarrow$ $\mathbb{X I}(\mathbf{X})$, defined by the formula $\tau_{\mathbf{X}}(x)=\varepsilon_{\mathbf{X}}(x)$, is a natural dual transformation between 
the functor $\mathbb{X I}$ and the functor $\operatorname{Id}_{\mathcal{X}}$, where $\varepsilon_{\mathbf{X}}$ is the natural isomorphism from $\mathbf{X}$ to $\operatorname{St}(\operatorname{Clop}(\mathbf{X}))$.

\section{Monadic Boolean Closure of A monadic implication AlgeBra}

3.1. Monadic implication algebras. A monadic implication algebra is an algebra $\mathbf{A}=$ $\langle A, \rightarrow, \forall, 1\rangle$ of type $(2,1,0)$ such that $\langle A, \rightarrow\rangle$ is an implication algebra and $\forall$ satisfies

(M1) $\forall 1=1$,

(M2) $\forall x \rightarrow x=1$,

(M3) $\forall((x \rightarrow \forall y) \rightarrow \forall y)=(\forall x \rightarrow \forall y) \rightarrow \forall y$,

(M4) $\forall(x \rightarrow y) \rightarrow(\forall x \rightarrow \forall y)=1$.

Since $x \vee y=(x \rightarrow y) \rightarrow y$, then (M3) can be written as $\forall(x \vee \forall y)=\forall x \vee \forall y$.

The following properties hold in every monadic implication algebra $\mathbf{A}([1])$ :

(M5) $\forall \forall x=\forall x$,

(M6) if $x \leq y$ then $\forall x \leq \forall y$,

(M7) if $x=\forall x$ and $y=\forall y$ then $x \rightarrow y=\forall(x \rightarrow y)$.

Let $\forall A=\{\forall x: x \in A\}$, then from (M1), (M5) and (M7), it follows that $\forall \mathbf{A}$ is an implication subalgebra of $\mathbf{A}$.

If $\mathbf{A}$ is a monadic implication algebra with least element 0, we know that $\mathbf{A}$ is a Boolean algebra. If we define $\exists x=\neg \forall \neg x$ then $\langle A, \wedge, \vee, \neg, \exists, 0,1\rangle$ is a monadic Boolean algebra $([9],[8])$.

A subset $D$ of a monadic implication algebra $\mathbf{A}$ is said to be an implication filter if $1 \in D$, and if $x, x \rightarrow y \in D$ then $y \in D$. An implication filter $D$ is said to be a monadic implication filter if $D$ satisfies that $\forall x \in D$ whenever $x \in D$.

It is known that the lattice of monadic implication filters of $\mathbf{A}$, the lattice of congruences of $\mathbf{A}$ and the lattice of implication filters of $\forall \mathbf{A}$ are all isomorphic [1, Section 2].

A non trivial monadic implication algebra $\mathbf{A}$ is simple if and only if the only monadic implication filters in $\mathbf{A}$ are $A$ and $\{1\}$. The following result will be needed.

Lemma 3.1. ([1]) A monadic implication algebra A is subdirectly irreducible (simple) if and only if $\mathbf{A}$ is a simple monadic Boolean algebra.

Every monadic implication algebra $\mathbf{A}$ is isomorphic to a monadic implication subalgebra $\mathbf{A}^{\prime}$ of $\mathbf{P}=\prod\{\mathbf{A} / D, D \in \mathrm{M}\}$, where $\mathrm{M}$ is the set of maximal monadic implication filters of $\mathbf{A}$. Moreover, for each $D \in \mathrm{M}, \mathbf{A} / D$ is a simple monadic implication algebra, and consequently, $\mathbf{A} / D$ is a simple monadic Boolean algebra. Thus, $\mathbf{P}$ is a monadic Boolean algebra. We identify $\mathbf{A}$ with $\mathbf{A}^{\prime}$.

Theorem 3.2. Let $\mathbf{A}$ be a monadic implication algebra, let $\mathbf{P}=\prod\{\mathbf{A} / D, D \in \mathbf{M}\}$, where $\mathrm{M}$ is the set of maximal monadic implication filters of $\mathbf{A}$ and let $B_{M}(\mathbf{A})$ be the monadic Boolean subalgebra generated by $A$ in $\mathbf{P}$. Then the monadic Boolean algebra $B_{M}(\mathbf{A})$ is equal 
to the Boolean algebra $B(\mathbf{A})$ generated by $A$ in $\mathbf{P}$. Moreover, $A$ is an increasing subset of $B_{M}(\mathbf{A})$.

Proof. Let us prove that $B(\mathbf{A})$ is closed under $\forall$. Let $b \in B(\mathbf{A})$. Then $b$ can be written as

$$
b=\bigwedge_{k=1}^{r}\left[\left(\bigvee_{i \in I_{k}} g_{i}\right) \vee\left(\bigvee_{j \in J_{k}} \neg g_{j}\right)\right]
$$

where $I_{k} \cap J_{k}=\emptyset$ for every $k, \bigcup_{k}\left(I_{k} \cup J_{k}\right) \neq \emptyset$ is a finite set and $g_{i}, g_{j} \in A$. So,

$$
\forall b=\bigwedge_{k=1}^{r} \forall\left[\left(\bigvee_{i \in I_{k}} g_{i}\right) \vee\left(\bigvee_{j \in J_{k}} \neg g_{j}\right)\right]
$$

If $I_{k} \neq \emptyset$ and $J_{k} \neq \emptyset$, then

$$
\left(\bigvee_{i \in I_{k}} g_{i}\right) \vee\left(\bigvee_{j \in J_{k}} \neg g_{j}\right)=\bigvee_{j \in J_{k}}\left(g_{j} \rightarrow \bigvee_{i \in I_{k}} g_{i}\right)=\bigvee_{j \in J_{k}} h_{j}
$$

where $h_{j} \in A$, since $\bigvee_{i \in I_{k}} g_{i} \in A$. Thus, $\forall\left[\left(\bigvee_{i \in I_{k}} g_{i}\right) \vee\left(\bigvee_{j \in J_{k}} \neg g_{j}\right)\right]=a_{k} \in A$, that is, $\forall b=\bigwedge a_{k}, a_{k} \in A$. Thus $\forall b \in B(\mathbf{A})$.

Similarly, if $I_{k} \neq \emptyset$ and $J_{k}=\emptyset$.

If $I_{k}=\emptyset$, then $\forall\left(\bigvee_{j \in J_{k}} \neg g_{j}\right)=\forall\left(\neg \bigwedge_{j \in J_{k}} g_{j}\right)=\neg \exists\left(\bigwedge_{j \in J_{k}} g_{j}\right)$. Let us see that $\exists\left(\bigwedge_{j \in J_{k}} g_{j}\right) \in B(\mathbf{A})$. We know that $\exists x=\forall(x \rightarrow \forall x) \rightarrow \forall x$. So,

$$
\begin{aligned}
\exists\left(\bigwedge_{j \in J_{k}} g_{j}\right) & =\forall\left[\left(\bigwedge_{j \in J_{k}} g_{j}\right) \rightarrow \forall\left(\bigwedge_{j \in J_{k}} g_{j}\right)\right] \rightarrow \forall\left(\bigwedge_{j \in J_{k}} g_{j}\right) \\
& =\forall\left[\left(\bigwedge_{j \in J_{k}} g_{j}\right) \rightarrow \bigwedge_{j \in J_{k}} \forall g_{j}\right] \rightarrow\left(\bigwedge_{j \in J_{k}} \forall g_{j}\right) \\
& \left.=\bigwedge_{j \in J_{k}}\left[\forall\left[\bigwedge_{j \in J_{k}} g_{j}\right) \rightarrow \bigwedge_{j \in J_{k}} \forall g_{j}\right] \rightarrow \forall g_{j}\right] .
\end{aligned}
$$

Let us see that $\forall\left[\left(\bigwedge_{j \in J_{k}} g_{j}\right) \rightarrow \bigwedge_{j \in J_{k}}\left(\forall g_{j}\right)\right] \rightarrow \forall g_{j} \in A$, for every $j \in J_{k}$. But,

$$
\begin{aligned}
& \forall\left[\left(\bigwedge_{j \in J_{k}} g_{j}\right) \rightarrow \bigwedge_{j \in J_{k}} \forall g_{j}\right] \rightarrow \forall g_{j}=\forall\left[\bigwedge_{j \in J_{k}}\left[\left(\bigwedge_{j \in J_{k}} g_{j}\right) \rightarrow \forall g_{j}\right]\right] \rightarrow \forall g_{j} \\
& =\forall\left[\bigwedge_{j \in J_{k}} \bigvee_{j \in J_{k}}\left(g_{j} \rightarrow \forall g_{j}\right)\right] \rightarrow \forall g_{j}=\left[\bigwedge_{j \in J_{k}}\left(\forall \bigvee_{j \in J_{k}}\left(g_{j} \rightarrow \forall g_{j}\right)\right)\right] \rightarrow \forall g_{j} \\
& =\bigvee_{j \in J_{k}}\left[\forall\left(\bigvee_{j \in J_{k}}\left(g_{j} \rightarrow \forall g_{j}\right)\right)\right] \rightarrow \forall g_{j} \in A .
\end{aligned}
$$


So, if $I_{k}=\emptyset$ then $\exists\left(\bigwedge_{i \in J_{k}} g_{i}\right) \in B(\mathbf{A})$. Therefore $\forall b \in B(\mathbf{A})$.

Now we have that $A$ is increasing in $B_{M}(\mathbf{A})=B(\mathbf{A})$ and closed under $\forall$. Then $\mathbf{A}$ is a union of monadic filters of $B_{M}(\mathbf{A})$. Consider now the monadic filter $F_{M}(\mathbf{A})$ generated by $A$ in $B_{M}(\mathbf{A})$. As $F_{M}(\mathbf{A})=F(\forall \mathbf{A})$, and since $\forall A \subseteq A$, it is easy to prove that $F_{M}(\mathbf{A})=F(\mathbf{A})$. Then the following result is immediate.

Lemma 3.3. Let $\mathbf{A}$ be an monadic implication algebra and let $F_{M}(\mathbf{A})$ be the monadic filter generated by $A$ in $B_{M}(\mathbf{A})$. An element $b \in B_{M}(\mathbf{A})$ is in $F_{M}(\mathbf{A})$ if and only if $b=\bigwedge_{i=1}^{n} a_{i}$, where $a_{i} \in A$.

3.2. Monadic Boolean closure. The aim of this subsection is to give for any monadic implication algebra $\mathbf{A}$ the least, up to isomorphisms, monadic Boolean algebra in which $F_{M}(\mathbf{A})$ is an ultrafilter. We will call this algebra the monadic Boolean closure of $\mathbf{A}$ and we will denote it by $\operatorname{Bm}(\mathbf{A})$.

Since $B_{M}(\mathbf{A})=B(\mathbf{A})$ and $F_{M}(\mathbf{A})=F(\mathbf{A})$, the construction of $\mathbf{B m}(\mathbf{A})$ is the same as in the case of an implication algebra, that is,

$$
\mathbf{B m}(\mathbf{A})=\left\{\begin{array}{cl}
B_{M}(\mathbf{A}) & \text { if } F_{M}(\mathbf{A}) \neq B_{M}(\mathbf{A}) \\
B_{M}(\mathbf{A}) \times\{0,1\} & \text { if } F_{M}(\mathbf{A})=B_{M}(\mathbf{A})
\end{array} .\right.
$$

Note also that if $\mathbf{B m}(\mathbf{A})=B_{M}(\mathbf{A}) \times\{0,1\}$ then $\exists$ is pointwise defined. The following theorem is the main result of this section.

Theorem 3.4. Let $\mathbf{A}$ be a monadic implication algebra, then there is a monadic Boolean algebra $\mathbf{B m}(\mathbf{A})$ such that,

(1) $A$ is an increasing subset of $\mathbf{B m}(\mathbf{A})$ and $F_{M}(\mathbf{A})$ is a monadic ultrafilter of $\mathbf{B m}(\mathbf{A})$ such that $\mathbf{B m}(\mathbf{A}) / F_{M}(\mathbf{A}) \cong\{0,1\}$,

(2) if $\mathbf{B}$ is a monadic Boolean algebra and $h: \mathbf{A} \rightarrow \mathbf{B}$ is a $\{\rightarrow, \forall\}$-homomorphism such that $h[\mathbf{A}]$ has the fmp in $\mathbf{B}$, then there is a monadic homomorphism $\hat{h}: \mathbf{B m}(\mathbf{A}) \rightarrow \mathbf{B}$ such that $\hat{h} \uparrow A=h$, that is, the following diagram commutes

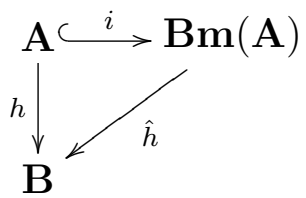

Proof. The condition (1) has already been proved.

For (2), let us consider $b \in \mathbf{B m}(\mathbf{A})$. If $b \in F(\mathbf{A})$, then, from Lemma 3.3, there are $a_{1}, \ldots, a_{n} \in A$, such that $b=\bigwedge_{i=1}^{n} a_{i}$ and we take $\hat{h}(b)=\bigwedge_{i=1}^{n} h\left(a_{i}\right)$. If $b \notin F(\mathbf{A})$, then we take $\hat{h}(b)=\neg \hat{h}(\neg b)$. From [3, Theorem 2.2], we know that $\hat{h}$ preserves $\rightarrow$ and $\hat{h}(0)=0$. We only need to prove that $\hat{h}(\forall b)=\forall \hat{h}(b)$. Suppose first that $b \in F(\mathbf{A})$. Then $b=\bigwedge_{i=1}^{n} a_{i}$, where $a_{i} \in A$, for every $i$. Hence, $\forall b=\bigwedge_{i=1}^{n} \forall a_{i}$ yielding $\hat{h}(\forall b)=$ $\bigwedge_{i=1}^{n} h\left(\forall a_{i}\right)=\bigwedge_{i=1}^{n} \forall h\left(a_{i}\right)=\forall\left(\bigwedge_{i=1}^{n} h\left(a_{i}\right)\right)=\forall \hat{h}(b)$. Now suppose that $b \notin F(\mathbf{A})$. Thus, $\hat{h}(\forall b)=\hat{h}(\neg \exists \neg b)=\neg \hat{h}(\exists \neg b)$. Observe that $\exists \neg b=\forall(\neg b \rightarrow \forall \neg b) \rightarrow \forall \neg b$. Moreover, $\hat{h}$ 
preserves $\rightarrow$ and $\neg b \in F(A)$, so $\neg \hat{h}(\exists \neg b)=\neg \exists \hat{h}(\neg b)=\neg \exists \neg \hat{h}(b)=\forall \hat{h}(b)$. We have proved that $\hat{h}$ is a monadic homomorphism.

As a consequence, we have the following.

Corollary 3.5. Let $\mathrm{M}(\mathbf{A})$ be the family of all maximal elements in the set of all monadic filters of $\mathbf{B m}(\mathbf{A})$ contained in the monadic implication algebra $\mathbf{A}$, ordered by inclusion. Then we have

(a) $A=\bigcup_{F \in \mathrm{M}(\mathbf{A})} F$,

(b) $\mathrm{M}(\mathbf{A})$ is an antichain,

(c) If $M$ is a monadic filter of $\mathbf{B m}(\mathbf{A})$ that is contained in $A$, then there is a filter $F \in \mathrm{M}(\mathbf{A})$ such that $M \subseteq F$.

Proof. (a) Since $\mathbf{A}$ is increasing in $B_{M}(\mathbf{A})$ and $B_{M}(\mathbf{A})$ is an ultrafilter of $\mathbf{B m}(\mathbf{A})$, then $\mathbf{A}$ is increasing in $\mathbf{B m}(\mathbf{A})$. In addition, $\mathbf{A}$ is closed under $\forall$. Since every increasing set $V$ in a Boolean algebra is a union of the filters contained in $V$, then $A$ is the union of the monadic filters of $\mathbf{B m}(\mathbf{A})$ contained in $A$. From Zorn's Lemma, $\mathbf{M}(\mathbf{A}) \neq \emptyset$. Thus $A=\bigcup_{F \in \mathrm{M}(\mathbf{A})} F$.

(b) It follows from the definition of $\mathrm{M}(\mathbf{A})$.

(c) It is an immediate consequence of (a).

The next result states that the monadic Boolean closure of a monadic implication algebra preserves homomorphisms, and it is unique, up to isomorphism.

Corollary 3.6. Let $h: \mathbf{A}_{1} \rightarrow \mathbf{A}_{2}$ be a monadic implication homomorphism. Then there is a monadic Boolean homomorphism $\hat{h}: \mathbf{B m}\left(\mathbf{A}_{1}\right) \rightarrow \mathbf{B m}\left(\mathbf{A}_{2}\right)$ such that $\hat{h} \uparrow \mathbf{A}_{1}=h$ and $\hat{h}^{-1}\left[F\left(\mathbf{A}_{2}\right)\right]=F\left(\mathbf{A}_{1}\right)$. In addition, if $h$ is an isomorphism then $\hat{h}$ is also an isomorphism.

Proof. From $h: \mathbf{A}_{1} \rightarrow \mathbf{A}_{2}$ and $A_{2} \subseteq \mathbf{B m}\left(\mathbf{A}_{2}\right)$ then $h: \mathbf{A}_{1} \rightarrow \mathbf{B m}\left(\mathbf{A}_{2}\right)$. Thus, by Theorem 3.4, there exists a monadic homomorfism $\hat{h}: \mathbf{B m}\left(\mathbf{A}_{1}\right) \rightarrow \mathbf{B m}\left(\mathbf{A}_{2}\right)$ such that $\hat{h} \uparrow \mathbf{A}_{1}=h$. From the definition of $\hat{h}$ and from the fact that $\hat{h}^{-1}\left[F\left(\mathbf{A}_{2}\right)\right]$ is an ultrafilter of $\mathbf{B m}\left(\mathbf{A}_{1}\right)$, we have that $\hat{h}^{-1}\left[F\left(\mathbf{A}_{2}\right)\right]=F\left(\mathbf{A}_{1}\right)$.

\section{MONADiC IMPLiCATiOn SPACES}

The results of the previous section will allow us to associate to any monadic implication algebra a topological space endowed with certain distinguished objects.

Observe that if $\mathbf{A}$ is a monadic implication algebra and $\mathcal{C}(\mathbf{A})=\left\{C_{F}: F \in \mathbf{M}(\mathbf{A})\right\}$, then $\mathbb{X}_{M}(\mathbf{A})=\left(\operatorname{St}(\mathbf{B m}(\mathbf{A})), R_{\exists}, F_{M}(\mathbf{A}), \mathcal{C}(\mathbf{A})\right)$ has the following properties:

- $\left(\operatorname{St}(\mathbf{B m}(\mathbf{A})), R_{\exists}\right)$ is a $\mathbf{B}$-space (Theorem 3.4),

- $F_{M}(\mathbf{A})$ is an element of $\operatorname{St}(\operatorname{Bm}(\mathbf{A}))$ (Theorem 3.4),

- $\mathcal{C}(\mathbf{A})$ is an antichain, with respect to inclusion, of closed subsets of $\operatorname{St}(\mathbf{B m}(\mathbf{A}))$ such that $R C=C$, for every $C \in \mathcal{C}(\mathbf{A})$, and $\cap \mathcal{C}=\left\{F_{M}(\mathbf{A})\right\}$ (Corollary 3.5). 
- if $C$ is a closed subset of $\operatorname{St}(\mathbf{B m}(\mathbf{A}))$ such that $R C=C$ and such that for every $N \in \operatorname{Clop}(X), C \subseteq N$ implies $D \subseteq N$ for some $D \in \mathcal{C}(\mathbf{A})$, then there is $D^{\prime} \in \mathcal{C}(\mathbf{A})$ such that $D^{\prime} \subseteq C$ (Corollary 3.5 and Definition $2.4(\mathrm{E} 4)$ ).

This motivates the following definition.

Definition 4.1. A monadic implication space is a 4-tuple $(\mathbf{X}, R, u, \mathcal{C})$ such that

(1) $(\mathbf{X}, R)$ is a $\mathbf{B}$-space,

(2) $u$ is a fixed element of $\mathbf{X}$,

$(3) \mathcal{C}$ is an antichain, with respect to inclusion, of closed sets of $\mathbf{X}$ such that $R C=C$, for every $C \in \mathcal{C}$ and $\bigcap \mathcal{C}=\{u\}$,

(4) if $C$ is a closed subset of $\mathbf{X}$ such that $R C=C$ and such that for every $N \in \operatorname{Clop}(\mathbf{X})$, $C \subseteq N$ implies $D \subseteq N$ for some $D \in \mathcal{C}$, then there is $D^{\prime} \in \mathcal{C}$ such that $D^{\prime} \subseteq C$.

From the above, we know that if $\mathbf{A}$ is a monadic implication algebra then $\mathbb{X}_{M}(\mathbf{A})$ is a monadic implication space.

Now we are going to define the notion of a morphism between two monadic implication spaces. For that, consider a homomorphism $f: \mathbf{A}_{1} \rightarrow \mathbf{A}_{2}$ of monadic implication algebras, and let $\left.\mathbb{X}_{M}(f)=\operatorname{St}(\hat{f}): \operatorname{St}\left(\mathbf{B m}\left(\mathbf{A}_{2}\right)\right)\right) \rightarrow \operatorname{St}\left(\mathbf{B m}\left(\mathbf{A}_{1}\right)\right)$ defined by $\operatorname{St}(\hat{f})(U)=\hat{f}^{-1}(U)$. We know that the following conditions are satisfied:

- $\mathbb{X}_{M}(f)$ is a B-morphism (see Definition 2.2).

- $\mathbb{X}_{M}(f)\left(F\left(\mathbf{A}_{2}\right)\right)=F\left(\mathbf{A}_{1}\right)$ (see Theorem 3.4).

Lemma 4.2. Let $\mathbf{A}_{1}, \mathbf{A}_{2}$ be monadic implication algebras and let $F \in \mathbf{M}\left(\mathbf{A}_{1}\right)$. Then there exists $H \in \mathrm{M}\left(\mathbf{A}_{2}\right)$ such that $C_{H} \subseteq \mathrm{St}(\hat{f})^{-1}\left[C_{F}\right]$.

Proof. Recall that $\operatorname{St}(\hat{f})^{-1}\left[C_{F}\right]=C_{F(f[F])}$ and $F(f[F]) \subseteq \mathbf{A}_{2},([3])$. We only need to prove that $F(f[F])$ is monadic. For that, let $b \in F(f[F])$ and $a_{i} \in F$ such that $b \geq \bigwedge_{i=1}^{n} f\left(a_{i}\right)$. Since $f$ preserves $\forall$, we have

$$
\forall b \geq \forall\left(\bigwedge_{i=1}^{n} f\left(a_{i}\right)\right)=\bigwedge_{i=1}^{n} \forall f\left(a_{i}\right)=\bigwedge_{i=1}^{n} f\left(\forall a_{i}\right) .
$$

Since $F$ is monadic, then $\forall a_{i} \in F$. From the maximality of the elements of $\mathrm{M}\left(\mathbf{A}_{2}\right)$, there exists $H \in \mathrm{M}\left(\mathbf{A}_{2}\right)$ such that $F(f[F]) \subseteq H$ and thus $C_{H} \subseteq C_{F(f[F])}$.

The above results suggest the notion of morphism between two monadic implication spaces.

Definition 4.3. Let $\left(\mathbf{X}_{1}, R_{1}, u_{1}, \mathcal{C}_{1}\right)$ and $\left(\mathbf{X}_{2}, R_{2}, u_{2}, \mathcal{C}_{2}\right)$ be two monadic implication spaces. $A \operatorname{map} f: X_{1} \rightarrow X_{2}$ is $i \mathbf{B}$-continuous if

(1) $f$ is a $\mathbf{B}$-morphism,

(2) $f$ is i-continuous.

If $f$ is a homeomorphism such that its inverse is also $i \mathbf{B}$-continuous then we say that $f$ is an $i$ B-homeomorphism. From [3, cor 3.2] and [6] we have the following. 
Corollary 4.4. If $f:\left(\mathbf{X}_{1}, R_{1}, u_{1}, C_{1}\right) \rightarrow\left(\mathbf{X}_{2}, R_{2}, u_{2}, C_{2}\right)$ is an $i \mathbf{B}$-morphism such that $f$ is a homeomorphism then, $f$ is an $i \mathbf{B}$-homeomorphism if and only if the following conditions are satisfied

- for all $D \in C_{1}$ we have that $f[D] \in C_{2}$,

- $x R_{1} y$ if and only if $f(x) R_{2} f(y)$.

\section{The NATURAL DUAL EQUiVAlEnCE}

We will denote by $\mathcal{M I}$ the category whose objects are monadic implication algebras and whose morphisms are monadic homomorphisms, and by $\mathcal{M E}$ the category of monadic implication spaces and $i \mathbf{B}$-morphisms. In this section, we prove that there is a natural dual equivalence between these categories.

Correspondence of objects. We have already proved that if $\mathbf{A}$ is a monadic implication algebra then $\mathbb{X}_{M}(\mathbf{A})=\left(\operatorname{St}(\mathbf{B m}(\mathbf{A})), R_{\exists}, F(\mathbf{A}), \mathcal{C}(\mathbf{A})\right)$ is a monadic implication space. Let $(\mathbf{X}, R, u, \mathcal{C})$ be a monadic implication space. Consider the implication algebra

$$
\mathbb{I}_{M}(\mathbf{X})=\{N \in \operatorname{Clop}(\mathbf{X}): C \subseteq N \text {, for some } C \in \mathcal{C}\} \text {. }
$$

We know that $\left\langle\operatorname{Clop}(\mathbf{X}), \exists_{R}\right\rangle$ is a monadic Boolean algebra. Let us prove that $\mathbb{I}_{M}(\mathbf{X})$ is a monadic implication algebra where $\forall N=\neg \exists_{R} \neg N=\mathcal{C} R \mathcal{C} N$. Observe that $\mathbb{I}_{M}(\mathbf{X})=\mathbb{I}(\mathbf{X})$ and then, $\mathbb{I}_{M}(\mathbf{X})$ is an implication algebra. Thus we have to prove that $\forall N \in \mathbb{I}(\mathbf{X})$, for every $N \in \mathbb{I}(\mathbf{X})$. Indeed, if $N \in \mathbb{I}_{M}(\mathbf{X})$, then there exists $C \in \mathcal{C}$ such that $C \subseteq N$. Thus, $R \mathcal{C} N \subseteq R \mathcal{C} C=\mathcal{C} R C=\mathcal{C} C$. Then, $C \subseteq \forall N$.

Observation 5.1. $\mathbb{I}_{M}(\mathbf{X})=\bigcup_{c \in \mathcal{C}} F_{C}$, where $F_{C}=\{N \in \operatorname{Clop}(\mathbf{X}): C \subseteq N\}$ and also $F\left(\mathbb{I}_{M}(\mathbf{X})\right)=\{N \in \operatorname{Clop}(\mathbf{X}): u \in N\}=\varepsilon_{\mathbf{X}}(U), \operatorname{Bm}\left(\mathbb{I}_{M}(\mathbf{X})\right)=\operatorname{Clop}(\mathbf{X})$ and $\mathbf{M}\left(\mathbb{I}_{M}(\mathbf{X})\right)=$ $\left\{F_{C}: C \in \mathcal{C}\right\}$.

Correspondence of morphisms. Let $f: \mathbf{A}_{1} \rightarrow \mathbf{A}_{2}$ be a homomorphism of monadic implication algebras and consider $\operatorname{St}(\hat{f}):\left(\operatorname{St}\left(\operatorname{Bom}\left(\mathbf{A}_{2}\right)\right), R_{\exists_{2}}\right) \rightarrow\left(\operatorname{St}\left(\operatorname{Bom}\left(\mathbf{A}_{1}\right)\right), R_{\exists_{1}}\right)$ as previously defined. We have seen that $\mathbb{X}_{M}(f)=\operatorname{St}(\hat{f})$ is $i \mathbf{B}$-continuous. If $h: X_{1} \rightarrow X_{2}$ is $i \mathbf{B}$-continuous, then $\mathbb{I}_{M}(h)=\operatorname{Clop}(h) \uparrow \mathbb{I}_{M}\left(\mathbf{X}_{2}\right): \mathbb{I}_{M}\left(\mathbf{X}_{2}\right) \rightarrow \mathbb{I}_{M}\left(\mathbf{X}_{1}\right)$ defines a monadic homomorphism of monadic implication algebras. Moreover, if $h$ is an $i \mathbf{B}$-homeomorphism then $\mathbb{I}_{M}(h)$ is an isomorphism (see [3, Lemma 3.5]).

Natural equivalence. From Corollary 3.6, and the above, we have the following theorem.

Theorem 5.2. The correspondence $\mathbb{X}_{M}: \mathcal{M I} \rightsquigarrow \mathcal{M E}$ defined by

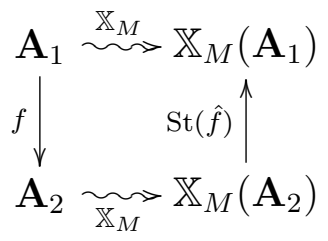

is a contravariant functor.

The correspondence $\mathbb{I}_{M}: \mathcal{M E} \rightsquigarrow \mathcal{M I}$ defined by 




is a contravariant functor.

Proof. We have already proved that $\mathbb{X}_{M}$ and $\mathbb{I}_{M}$ transform objects into objects and morphisms into morphisms. It is straightforward to prove that $\mathbb{X}_{M}$ and $\mathbb{I}_{M}$ are compatible with composition and transform the identity into the identity.

Theorem 5.3. The functors $\mathbb{I}_{M}$ and $\mathbb{X}_{M}$ define a dual equivalence between the categories $\mathcal{M I}$ and $\mathcal{M E}$. That is, if $\mathbf{A}$ is a monadic implication algebra,

$$
\sigma_{\mathbf{A}}: \mathbf{A} \rightarrow \mathbb{I}_{M} \mathbb{X}_{M}(\mathbf{A})
$$

defined by $\sigma_{\mathbf{A}}(a)=N_{a}$, is a natural transformation from the functor $\mathbb{I}_{M} \mathbb{X}_{M}$ to the identity functor $I d_{\mathcal{M I}}$. For every monadic implication space $(\mathbf{X}, R, u, C)$, the map

$$
\tau_{\mathbf{X}}: \mathbf{X} \rightarrow \mathbb{X}_{M} \mathbb{I}_{M}(\mathbf{X})
$$

defined by $\tau_{\mathbf{X}}(x)=\varepsilon_{\mathbf{X}}(x)$, is a natural transformation from the functor $\mathbb{X}_{M} \mathbb{I}_{M}$ to the identity functor $I d_{\mathcal{M E}}$.

Proof. In order to prove that $\sigma_{\mathbf{A}}$ is a natural transformation we have to prove that the following diagram commutes:

$$
\begin{array}{ccc}
\mathbf{A} & \stackrel{\sigma_{\mathbf{A}}}{\rightarrow} & \mathbb{I}_{M} \mathbb{X}_{M}(\mathbf{A}) \\
f \downarrow & & \downarrow \mathbb{I}_{M} \mathbb{X}_{M}(f) \\
\mathbf{A}^{\prime} & \stackrel{\sigma_{\mathbf{A}^{\prime}}}{\rightarrow} & \mathbb{I}_{M} \mathbb{X}_{M}\left(\mathbf{A}^{\prime}\right)
\end{array}
$$

where $\mathbf{A}$ and $\mathbf{A}^{\prime}$ are monadic implication algebras and $f: \mathbf{A} \rightarrow \mathbf{A}^{\prime}$ is a monadic homomorphism.

Observe that $\sigma_{\mathbf{B m}(\mathbf{A})}$ is an isomorphism from the monadic Boolean algebra $\langle\mathbf{B m}(\mathbf{A}), \exists\rangle$ onto $\left\langle\operatorname{St}(\operatorname{Clop}(\mathbf{X})), R_{\exists_{R}}\right\rangle$, and then $\sigma_{\mathbf{A}}=\sigma_{\mathbf{B m}(\mathbf{A})} \uparrow_{\mathbf{A}}$. So, to see that $\sigma_{\mathbf{A}}$ is an isomorphism it is enough to prove that $\sigma_{\mathbf{B m}(\mathbf{A})}[\mathbf{A}]=\mathbb{I}_{M} \mathbb{X}_{M}(\mathbf{A})$.

If $a \in A$, then there exists $F \in \mathrm{M}(\mathbf{A})$ such that $a \in F$. So $C_{F} \subseteq N_{a}$, that is, $\sigma_{\mathbf{A}}(a)=N_{a} \in \mathbb{I}_{M} \mathbb{X}_{M}(\mathbf{A})$.

If $N \in \mathbb{I}_{M} \mathbb{X}_{M}(\mathbf{A})$, there exists $a \in \mathbf{B m}(\mathbf{A})$ such that $N=N_{a} \in \mathbb{I}_{M} \mathbb{X}_{M}(\mathbf{A})$. Thus there exists $F \in \mathrm{M}(\mathbf{A})$ such that $C_{F} \subseteq N_{a}$, that is, every ultrafilter of $\mathbf{B m}(\mathbf{A})$ containing $F$ also contains $a$. So $a \in F \subseteq A$.

Observe that

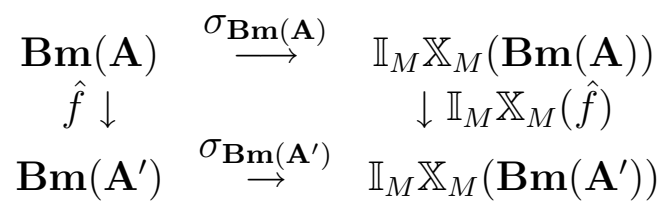


commutes (see Section 2.1, Theorem 3.4 and [11, Th. 8.2]), being that $\sigma_{\mathbf{B m}}$ is a natural transformation between the identity functor in the category of monadic Boolean algebras and the functor Clop St.

Then we have $\sigma_{\mathbf{A}}=\sigma_{\mathbf{B m}(\mathbf{A})} \uparrow_{\mathbf{A}}, \hat{f} \uparrow_{\mathbf{A}}=f$, and $\mathbb{I}_{M} \mathbb{X}_{M}(f)=\mathbb{I}_{M}(\operatorname{St}(\hat{f}))=\operatorname{Clop}\left\lceil_{\mathbb{I}_{M} \mathbb{X}_{M}(\mathbf{A})}\right.$

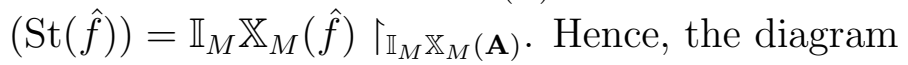

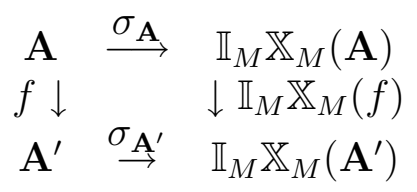

commutes. Thus $\sigma_{\mathbf{A}}$ gives a natural transformation.

The proof of the second part is analogous, and it follows from the definition of $\tau_{\mathbf{X}}$, Corollary 4.4 and Observation 5.1.

Acknowledgment. The authors would like to thank the referee for his/her helpful comments that improved the final version of this paper.

\section{REFERENCES}

[1] M. Abad, L. Monteiro, S. Savini and J. Seewald, Free Monadic Tarski Algebras, Algebra Universalis, 37(1997), 106-118.

[2] M. Abad, J. P. Díaz Varela amd M. Zander, Varieties and quasivarieties of monadic Tarski algebras, Sci. Math. Jpn., 568(3)(2002), 599-612.

[3] M. Abad, J. P. Díaz Varela and A. Torrens, Topological representation for implication algebras, Algebra Universalis, 52 (2004), 39-48.

[4] J. C. Abbott, Implicational algebras, Bull. Math. Soc. Sci. Math. R. S. Roumanie, 11(59) (1967), 3-23.

[5] S. Burris and H. P. Sankappanavar, A Course in Universal Algebra, Graduate texts in Mathematics 78, Springer Verlag, New York, 1981.

[6] R. Cignoli, Quantifiers on distributive lattices, Discrete Mathematics, 96 (1991), 183-197.

[7] P. R. Halmos, Algebraic logic. I. Monadic Boolean algebras, Compositio Math., 12 (1956), 217-249.

[8] P. R. Halmos, Free monadic algebras, Proc. Amer. Math. Soc., 10 (1959), 219-227.

[9] P. R. Halmos, Algebraic logic, Chelsea Publishing Co., New York, 1962.

[10] L. Iturrioz and A. Monteiro, Représentation des algèbres de Tarski monadiques, Preprint.

[11] S. Koppelberg, Handbook of Boolean Algebras, Elsevier Science Publishers B. V., (1989).

Departamento de Matemática, Universidad Nacional del Sur and Universidad Nacional del Comahue, Bahía Blanca y Neuquén

E-mail address: $\{$ imabad,crcima, usdiavar\}@criba.edu.ar 